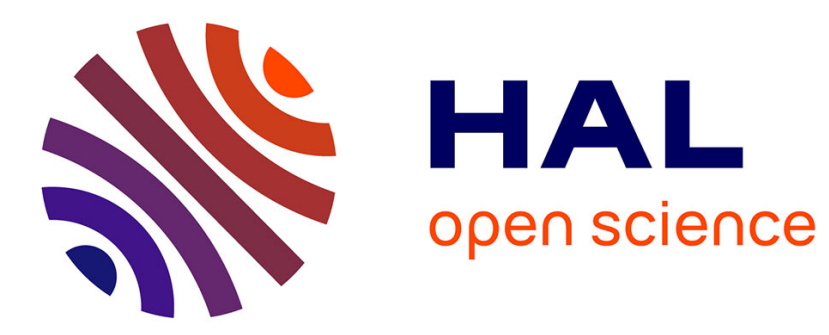

\title{
Terahertz waves for contactless control and imaging in aeronautics industry
}

A. Chopard, Q. Cassar, J. Bou-Sleiman, J.P. Guillet, M. Pan, J.B. Perraud, A. Susset, Patrick Mounaix

\section{- To cite this version:}

A. Chopard, Q. Cassar, J. Bou-Sleiman, J.P. Guillet, M. Pan, et al.. Terahertz waves for contactless control and imaging in aeronautics industry. NDT \& E International, 2021, 122, pp.102473. 10.1016/j.ndteint.2021.102473 . hal-03434613

\section{HAL Id: hal-03434613 \\ https://hal.science/hal-03434613}

Submitted on 25 Nov 2021

HAL is a multi-disciplinary open access archive for the deposit and dissemination of scientific research documents, whether they are published or not. The documents may come from teaching and research institutions in France or abroad, or from public or private research centers.
L'archive ouverte pluridisciplinaire HAL, est destinée au dépôt et à la diffusion de documents scientifiques de niveau recherche, publiés ou non, émanant des établissements d'enseignement et de recherche français ou étrangers, des laboratoires publics ou privés. 


\title{
Terahertz waves for contactless control and imaging in aeronautics industry
}

\author{
A. Chopard ${ }^{1,2}$, Q. Cassar ${ }^{1}$, J. Bou Sleiman ${ }^{3}$, J.P. Guillet ${ }^{1}$, M. Pan ${ }^{1}$, J.B. Perraud ${ }^{1}$, A. Susset ${ }^{3}$, P. \\ Mounaix ${ }^{1}$ \\ ${ }^{1}$ Laboratoire IMS-UMR CNRS 5218, Université Bordeaux, 33405, France \\ ${ }^{2}$ Lytid SAS - 8 Rue la Fontaine, 92120 Montrouge, France \\ ${ }^{3}$ R\&D-Vision - 64 rue Bourdignon 94100 Saint-Maur des Fossés, France
}

Elsevier use only: Received date here; revised date here; accepted date here

Article Info

Keywords:

Terahertz;

Imaging;

Multilayer painting;

FMCW radar;

Time domain

spectroscopy;
Abstract

The usability of terahertz systems for specific inspection tasks and imaging in the aeronautics industry is assessed. Especially, we demonstrate the suitability of frequency-modulated continuous-wave (FMCW) radars for health monitoring and see-through imaging. Additionally, terahertz timedomain data processing is performed for multi-layered paint structure characterization. FMCW radar principles are introduced. Available systems are described along with their benefits and limitations. Defect detection capabilities and progresses towards airplane covering see-through imaging are illustrated through FMCW experimental results on real samples. The suitability of FMCW radars as an advanced contactless non-destructive testing (NDT) tool for the aeronautics industry and maintenance services is demonstrated. A second application, based on terahertz time domain techniques, targets the characterisation of multi-layered painting structures through the assessment of dielectric properties and individual thickness of each layer deposited. Beside the review of extraction methods, the introduced new algorithm allows to derive a parametric transfer function, thus denoting the main contributions which give rise to the recorded terahertz electromagnetic field. Such a development pushes further the understanding and characterization of stratified structures by means of terahertz radiations and represents an indispensable tool to efficiently localise any deviation to the nominal painting stack in terms of thickness or dielectric properties. 


\section{Introduction}

Terahertz systems are demonstrating an increasing appeal through a wide panel of applicative fields thanks to their ability to provide contactless and non-destructive sensing solutions for in depth inspection. From security applications [1] to biomedical sensing [2-3] through non-destructive testing (NDT) [4-5] or even art inspection [6-7], specific integrations are demonstrating the multiple applicative capabilities of terahertz technologies.

The wide advances in this field in the recent years enabled the development of compact and cost-effective systems, driven by the increasing demand in dedicated complementary techniques applied to specific niche problematics [8]. Among those, complex materials integrity monitoring as well as penetrating imaging and sensing for NDT tasks is proving to be an ideal application fields for frequency modulated continuous-wave (FMCW) radars while an increasing demand in stratified structures inspection for the automotive and aerospace industries is being fulfilled with the implementations of terahertz time-domain spectroscopy (TDS) techniques.

\section{Radar imaging Capabilities for NDT}

The potential of FMCW sensing comes from its intrinsic benefits such as its high level of integration and compactness, its relatively low cost as well as the harmless character of the emitted radiations and their a priori high penetration capabilities in optically opaque materials. These arguments make FMCW a first-grade choice to complete the panel of tools allowing contactless inspection. Additionally, FMCW radars intrinsically provide information on the wave propagation delay and therefore straightly enables depth sensing. Hence, direct 3D imaging can be assessed [9][10], representing, along with the previously stated specificities, a non-negligible benefit in comparison with X-Rays imaging, low frequency continuous wave systems or thermographic or ultra-sound inspection methods. The detection and localization of damages, cracks, impact, delamination or inclusions are then achievable for materials that are commonly used in the aeronautics industry, such as glass fiber-based composite, honeycomb structures, polymers or ceramics either during fabrication or maintenance processes.

\subsection{FMCW radar architecture and principle}

FMCW radar have found several applicative purposes, from automotive anti-collision systems as precursors, to high-end NDT tools and have been developed on various technologies, mainly Si-based fully integrated devices [11] or III/V high frequency precision radars [12]. Despite the inherent benefits and drawbacks of each technology, their implementations nevertheless rely on similar architectures, described in Figure 1 (a). The first stage of the system, working at low frequency, features a voltage control oscillator (VCO), eventually combined to a phase locked loop (PLL) for stabilization purposes, in order to generate fast saw tooth-like frequency sweep. The multipliers chain is then introduced for up-conversion to reach the desired working frequency band, typically centred around $100 \mathrm{GHz}, 150 \mathrm{GHz}$, $300 \mathrm{GHz}$ or even up to $600 \mathrm{GHz}$. Nevertheless, the higher the frequency band, the lower the emitted power due to conversion losses. This high frequency sweep, considered as a reference signal, is then emitted towards the target (plain red Figure 1 (b)). By modulating the frequency in such a way, it is equivalent as putting a unique "time stamp" on the emitted wave at every instant.

The reflected sweep, delayed by the back and forth propagation through and into the target, (dotted orange Figure 1 (b)) is then collected and guided towards the detection mixer, thanks to a directional coupler, where a beating electric field is generated via the multiplication of this return sweep with the reference electromagnetic wave or one of its lower harmonics, due to their slight frequency difference. Collected by a data acquisition unit (DAQ), this beating signal displays a main frequency $f_{\text {mixer }}$, proportional to the propagation delay and so the distance to the object (see Equation (1)). From this formulation, the longitudinal resolution $\delta_{\text {res }}$ is derived (see Equation (2)) and solely relies on the bandwidth of the radar transceiver.
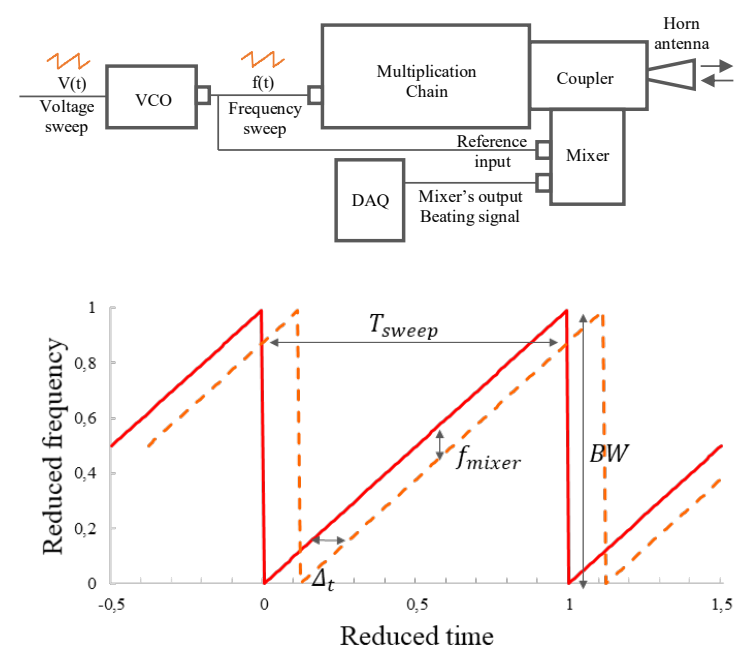

Figure 1: (a) Typical radar unit architecture (b), frequency sweep sensing scheme for FMCW measurement

$$
\begin{gathered}
d=\frac{c_{0} \Delta_{t}}{2 n}=\frac{c_{0} f_{\text {mixer }}}{2 n} \cdot \frac{T_{\text {sweep }}}{B W}, \\
\delta_{\text {res }}=\frac{c_{0}}{2 n B W},
\end{gathered}
$$

where $c_{0}$ is the speed of light in vacuum, $\Delta_{t}$, the back and forth propagation-induced time delay, $n$, the refracting index of the propagation media at the working frequency, $B W$, the frequency bandwidth, $f_{\text {mixer }}$, the beating frequency and $T_{\text {sweep }}$, the sweep period. 
For such radar unit architectures, the imaging capabilities are not intrinsic to those implementations due to the emission of a diverging beam from the antenna. The imaging process [4],[13] can nevertheless be performed through synthetic aperture radar reconstruction or more classically thanks to a focused raster scanning implementation. Due to the complexity of the inspected objects for see through imaging problematics, the latter solution is chosen and a quasioptical coupling system for focalized beam shaping is implemented with 2 Teflon ${ }^{\circledR}$ lenses, as displayed in Figure 2. Such an integration enables a simplified optimisation of the beam focus on the object of interest, beyond an eventual extra partially transparent cover, to reach the optimum returned power and resolution.

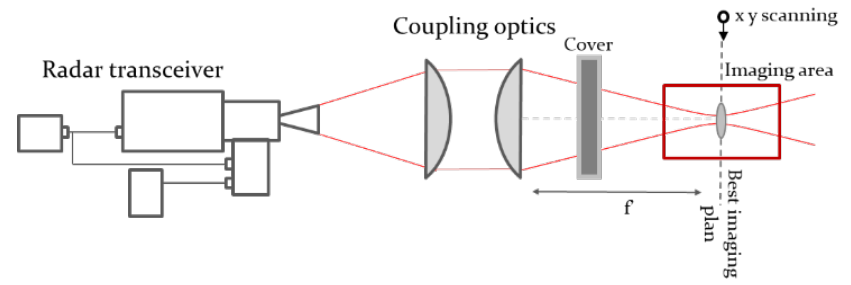

Figure 2: Optical configuration diagram for radar imaging in reflection mode with intermediate covering panel.

The Gaussian beam model, provides the direct relation between the beam waist diameter $\omega_{0}$, hence the resolution limit, and the focal length, $f$ ', of the focusing lens, as follows:

$$
\omega_{0}=\frac{4 \lambda f^{\prime}}{\pi \mathrm{D}}
$$

with $D$ the diameter of the lens, and $\lambda$ the wavelength related to the emitting source.

Therefore, for such a transceiver implementation, the resolution relies on the frequency band for the longitudinal resolution, as well as the optical configuration through the beam shaping system for the lateral resolution. At given working frequency, a trade-off between achievable lateral resolution and optimum imaging distance needs to be considered. Moreover, the adequate frequency band selection thus represents one of the key optimization since higher frequency bands would ensure an improved lateral resolution (see Equation 3) and would allow larger achievable bandwidth, therefore, providing enhanced longitudinal resolution as well (see Equation 2). Nevertheless, a wide range of aeronautics related materials remains sufficiently transparent for millimeter wave radiation, but commonly displays a significant increase in absorption with frequency[14], therefore resulting as well in a trade-off between material penetrability and targeted resolution depending on the defect size.

\subsection{D Imaging results toward see-through applications}

A wide range of samples based on glass-fiber and reinforced plastic have been inspected using a SynView imaging system in reflection or transmission mode and have been evaluated using $100 \mathrm{GHz}$ and $300 \mathrm{GHz}$ radar transceivers, as displayed in Figure 3 for health monitoring applications. Those images, are resulting from processed 2D radar scans, providing 3D inspection data thanks to the depth sensing capability of the FMCW method, with data retrieval in the object's best imaging plan. The high measurement rate of FMCW radar units, typically in the range of few $\mathrm{kHz}$, correlated with the millimeter achievable resolution, ensures an adequacy with the imaging cadencies expected for NDT applications, with a typical measurement time of a few minutes for such sample dimensions. As expected, higher lateral and longitudinal resolutions are achieved with the $300 \mathrm{GHz}$ scan while the lower measurement dynamic range of the systems as well as the reduced material penetrability represents a large drawback for such inspection tasks. Nevertheless, intentional foreign inclusions remain discernible for both measurements. On the contrary, carbon fiber-doped samples, displays high reflectivity over the terahertz range, preventing in-depth or see-through inspection (maximum of one carbon fibre layer can be screened) but allowing topographic inspection or coatings analysis.
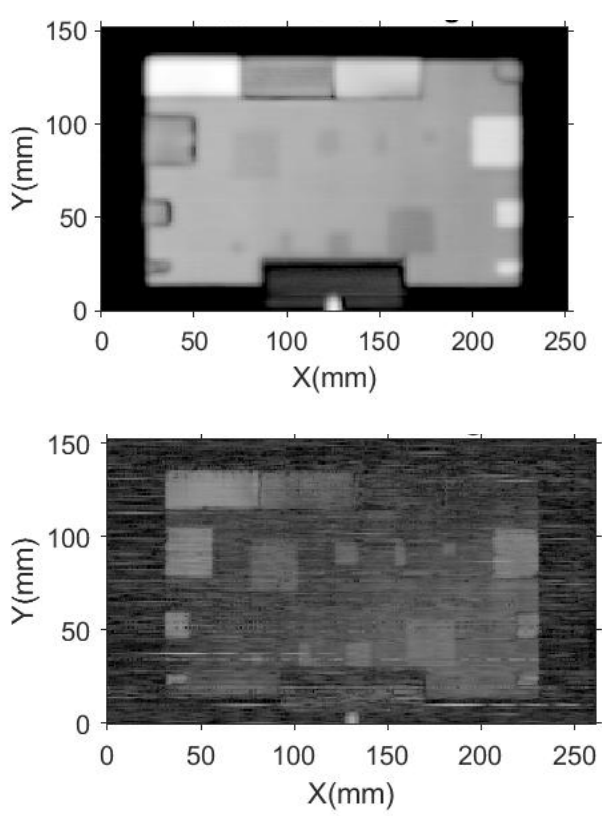

(b)

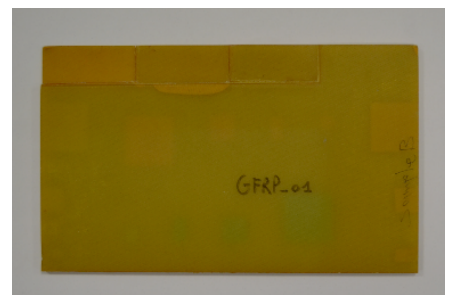

(a) $100 \mathrm{GHz}$ (b) and $300 \mathrm{GHz}$ radar scan in reflection mode of a glass fiber composite test sample displaying inclusions, (c) picture of the corresponding sample. 
Focusing on the see-through imaging problematics nevertheless brings to light few challenges. Targeting in-situ direct inspections while avoiding cover dismantlement, the reflection geometry represents the only adequate sensing implementation. Due to lowered material absorption levels at low frequencies, the $100 \mathrm{GHz}$ band radar transceiver turned out to be the most suitable in order to minimize the losses when passing back and forth through the fiber-glass panel while enabling objects sensing and guaranteeing adequate resolution of a few millimetres.

As a result, Figure 4 (a) displays the first demonstration of this system, in such a complex configuration of see-through plane cover imaging. A 3D animated representation, resulting from the $2 \mathrm{D}$ scan, of multiple reflective samples behind a plane cover, is available through the attached supplementary materials. The goal is to evaluate if we can properly detect and identify a metallic reflective object, such as a wrench, a tube or the sample holders. All the $1 / 2$ inch metallic post are also clearly imaged and resolved with a very good contrast. To push further the investigation, other objects that displays much lower reflectivity levels, such as a dry and moist sponges (Figure 4 (a) grey dotted box) held below this metallic wrench, are inserted, aiming to check the water content detection capability in case of crack or fissure inducing any fluid leakage. Clearly noticeable, a differentiation of the moisture level is achieved between the two sponges in presence and absence of water content.
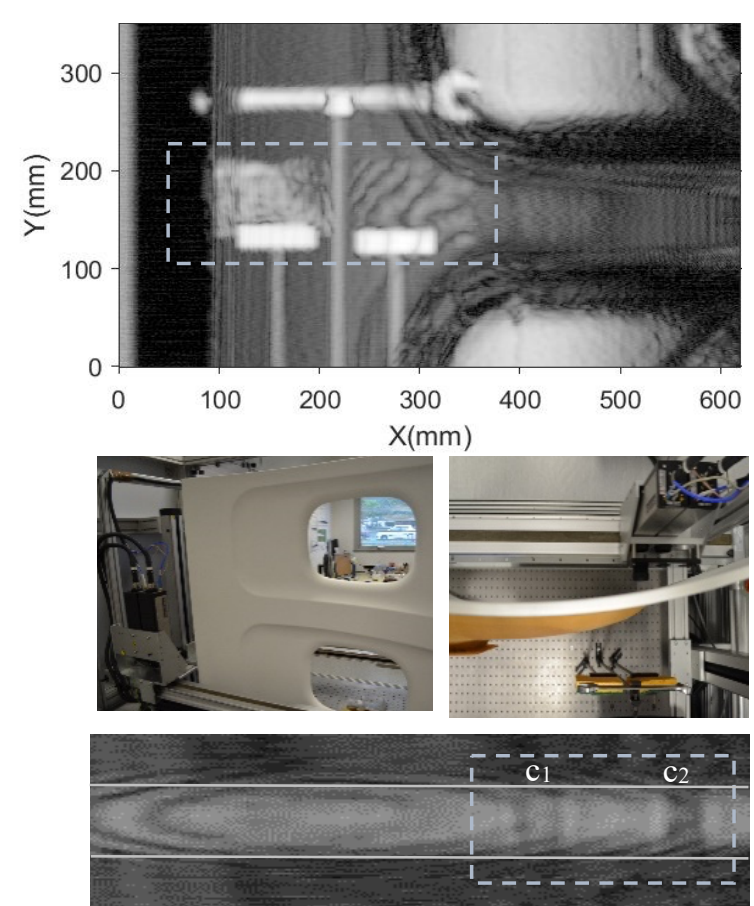

(a) $100 \mathrm{GHz}$ image of a wrench and sponges with their support through a plane covering panel (b) Synview imaging system with implemented plane cover (side view and top view) metallic samples and sponges are visible on the low right side (c) zoom on a $100 \mathrm{GHz}$ Image of an altered aluminum tube that displays $2 \mathrm{~mm}\left(c_{1}\right)$ and $5 \mathrm{~mm}\left(\mathrm{c}_{2}\right)$ cracks on its right part (dashed frame), through a plane covering panel.
Reminiscences of the panel geometry remains nevertheless discernible. We obtained useful images through the cover over distances up to $150 \mathrm{~mm}$ between the imaging area and the covering panel. Pushing further the tests, towards real case implementation, we demonstrated that the insertion of extra thermal insulation layers has no effect on the imaging capabilities thanks to their full transparency in the low frequency terahertz range (confirmed by terahertz spectroscopy). Beside simple object sensing and identification, Figure 4 (c) depicts the capability of see-through radar imaging for detection of defects and alterations, such as cracks or broken components, through a cover. For this implementation, a zoom on part of a $19 \mathrm{~mm}$ diameter aluminium tube (delimited by grey horizontal lines) displaying two controlled breaks ( $\mathrm{c}_{1}$ and $c_{2}$ in the grey dotted square box).

Finally, besides the many parameters impacting the sensing capabilities of radar units, such as the investigated feature's depth and size and their optical proprieties, the visualization of defects like foreign material, object, alterations or moisture contamination is made possible through a glass-fiber plane cover. A monitoring of the spatial location of objects of interests can moreover be performed thanks to the inherent depth sensing capabilities of radar imaging techniques.

The embedded phase information provided by the FMCW sensing process turns out to be an adequate tool for 3D imaging and in-depth sensing for a large scope of applicative cases of failure. Among those, the inspection of multi-layered polymers and foams structures for thickness inspection illustrates those capabilities [15]. With similar motives [16] and capabilities, but targeting scaled down structures, terahertz thickness inspection have been successfully implemented for micrometric stacked paint structures in the aeronautics and automotive industry with the use of time domain spectroscopy tools

\section{Thickness extraction and optical paths}

Although the use of FMCW radars has proven to be effective for contactless and non-destructive in-depth measurements for the aviation industry, complementary technologies whose operating frequency range remains within the terahertz band can address additional issues encountered by this industrial sector. That is notably the case of terahertz time-domain spectroscopy, primarily introduced in the field to control the thickness of aircraft coatings. The sensitivity of pulsed terahertz radiations to individual coatings forming a stratified structure was reported for different study cases, as in automotive, art science and obviously, aeronautics [17-20]. Indeed, when reaching a dielectric interface, a portion of the incident frequency-dependent terahertz electric field is transmitted through the following layer, while another portion 
is reflected back. Eventually, a time-of-flight analysis is conducted to evaluate the respective thicknesses.

Inverse electromagnetic problems are commonly performed to extract, from a recorded terahertz electric field the individual layer dielectric properties and thicknesses. Usually, it consists in minimizing an objective function that denotes the discrepancies between the experimental waveform and the ones computed from a set of candidate parameters that are aimed to impact the shape of the signal as the complex refractive index and the thickness. One can, non-exhaustively, distinguish three main different ways to proceed: (i) incremental Newtonian methods; (ii) full-volume test; (iii) dynamic stochastic strategy. In the following, each method is briefly explained and their main advantages and inconveniences are underlined.

\subsection{Incremental Newtonian methods}

The incremental Newtonian approach [21] consists in evaluating iteratively the curvature of the objective function based on the ratio of the gradient of this objective function over its Hessian. While such a process would make high progress initially, the speed of convergence decreases as it gets closer to the physical solution. Hence, it is suitable to set a threshold under which, the method ends.

\subsection{Full-volume test}

In comparison to the previous method, a full-volume test successively computes the objective function for all possible combinations of allowed-parameters. Therefore, the method is expected to converge to the physical solution. However, it is worth to note that the nominal number of calculations can be immense so that the process becomes cumbersome and would not, ultimately, be able to provide the physical parameters that compose the stack in a decent period of time.

\subsection{Dynamic stochastic strategy}

In comparison to the two previous methods, a stochastic process is aimed to randomly pick different combinations of parameters that are subsequently injected into the objective function. Two different approaches are possible to select the best parameters: (i) the test ends when a combination provides an objective function minimization that is greater than a predefined correlation threshold; (ii) the process terminates after a given number of shots and selects the combination that provides the smallest value of the objective function. As dealing with random selection processes, the convergence time of the first approach remains erratic and unpredictable. The later approach is accomplished within a fixed period of time but may not grasp the physical combination if the number of stochastic shots revealed to be too small. Note that in a similar fashion to the definition of convergence time for the first method, the number of required shots to find a valid combination cannot be predicted.
However, in both cases the probability to pick the valid combination increases as the number of allowed parameters decreases.

\subsection{Iterative tree algorithm (ITA)}

The aforementioned procedures are then commonly implemented to extract the physical parameters of each individual layers that constitute a stack. However, those methods gather indistinguishably the contributions of all the possible propagation paths through the paint stack, thus requiring a large computation power. The number of calculated paths could be gigantic while a precise knowledge of the most contributing optical paths would awfully minimize the quantity of computed routes and clarify the involved propagation processes. Furthermore, the derivation of a simplified parametric transfer function would drastically reduce the required computation power to minimize the objective function of an inverse electromagnetic formulation. A new algorithm monitoring the pulse subdivisions at dielectric interfaces has been to this end, developed [22]. The algorithm to be reported does not aim at evaluating the respective thicknesses of individual coatings but intends to establish a parametric transfer function, also referred as "reduced transfer function" in the following, which is derived from the analysis of individual optical route contribution to the responsive terahertz frequency-depend electric field. Ultimately, the so-called parametric transfer function can be used within the designed objective function to be minimized to extract, from one of the aforementioned property extraction methods, the thicknesses of coatings.

The iterative tree algorithm (ITA) classifies each portion of the signal as a function of the number of encountered dielectric interfaces instead of considering the signal as a whole. Additionally, it identifies the various optical paths involved for each contribution. The optical routes that contribute the most to the power are labelled and used to derive a parametric transfer function.

The central principle of the algorithm is to increment, at each encountered dielectric interface, a parameter $k$ denoting pulse subdivisions (Figure 5). Each propagating radiation is thus the combination of reflection and transmission processes involving surrounding dielectric layers, and occurring at the previous algorithm step, i.e. $k-1$.

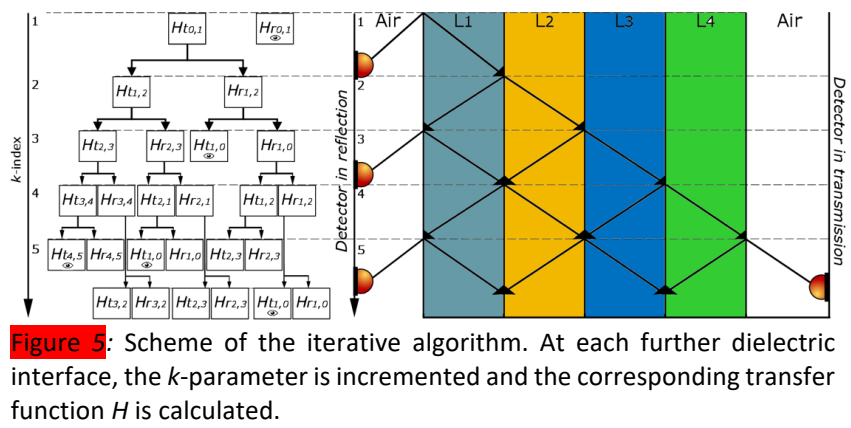


One has then the following equivalent mathematical description of the scheme:

$$
\left\{\begin{array}{l}
S_{r_{i, i+1}}^{k}=H_{r_{i, i+1}}\left(S_{r_{i, i-1}}^{k-1}+S_{t_{i-1, i}}^{k-1}\right) \\
S_{r_{i, i-1}}^{k}=H_{r_{i, i-1}}\left(S_{r_{i, i+1}}^{k-1}+S_{t_{i+1, i}}^{k-1}\right) \\
S_{t_{i, i+1}}^{k}=H_{t_{i, i+1}}\left(S_{r_{i, i-1}}^{k-1}+S_{t_{i-1, i}}^{k-1}\right) \\
S_{t_{i, i-1}}^{k}=H_{t_{i, i-1}}\left(S_{r_{i, i+1}}^{k-1}+S_{t_{i+1, i}}^{k-1}\right)
\end{array}\right.
$$

where $S_{r}^{k}$ and $S_{t}^{k}$ are the reflected and transmitted signals in frequency domain of the $k$-th iteration at each dielectric interface.

In order to pilot and to illustrate the iterative tree algorithm, an aeronautic stack sample on metallic substrate made of 4 layers of tens of microns has been extensively investigated in the $\mathrm{THz}$ domain and with conventional optical destructive methods for reference. The first step to achieve this investigation, was to carefully extract the dielectric properties of each individual painting material in isolation. To do so, the full-volume test reported in section 3.2 was followed. Each individual layer dielectric properties were then fitted to a single-pole Debye profile, as a consequence of a generalised thermal effect. The full stack was then reconstructed by means of equations (4) and its reconstruction iteratively processed is given in Figure 6.

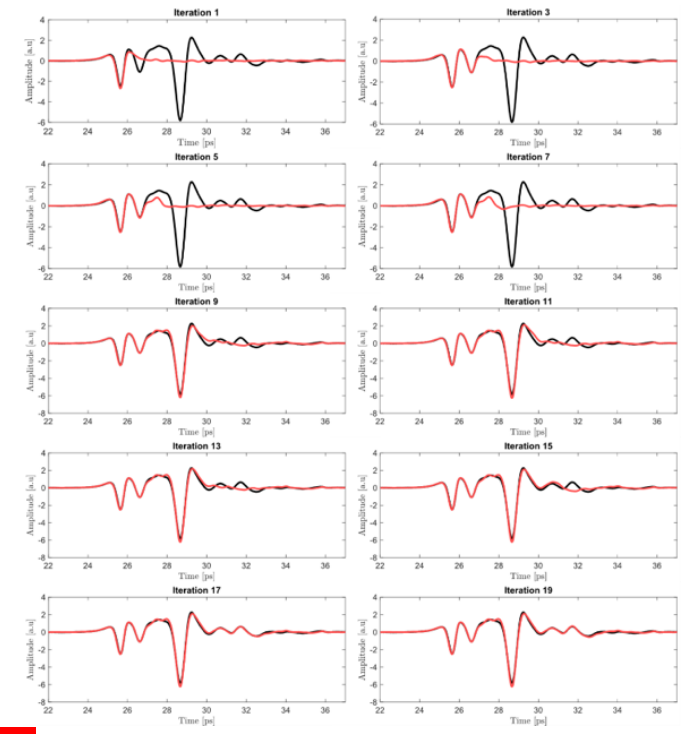

Figure 6: Iterative reconstruction (red) of the experimental waveform (black) for the aeronautic stack made of four layers, each of tens of microns.

Discrepancies between the computed waveform and the experimental one decreases when considering an increasing number of contributions until it reaches a threshold under which the error remains overall stable. The error metric, defined as the difference squared between the two waveforms, as a function of the number of iterations is given in Figure 7. The non-monotonous nature of the dependence reflects that time features are in majority due to the combination of several physical processes. Despite additional iterations, the error function does not reach zero. The presence of a non-zero asymptote may be attributed to various roots: the non-perfect extraction of individual dielectric profiles, experimental limits and additionally due to the fact that time features corresponding to further iterations are out of the time window in which the comparison metric is defined.

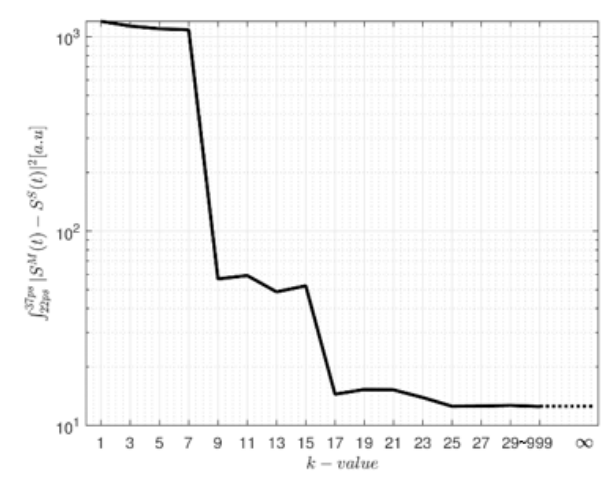

Figure 7: Profile of the integrated comparison metric between 22 ps and $37 \mathrm{ps}$ as a function of the number of iterations.

One has to keep in mind that such a reconstruction actually considers the whole possible optical routes within the stack. However, one can anticipate that all of these routes are not significantly contributing to the reflected signal. Thanks to the mathematical design of the algorithm, it is possible to analyse and quantify the contribution of each optical path individually and consequently to sort them as a function of their respective contributions to the responsive waveform.
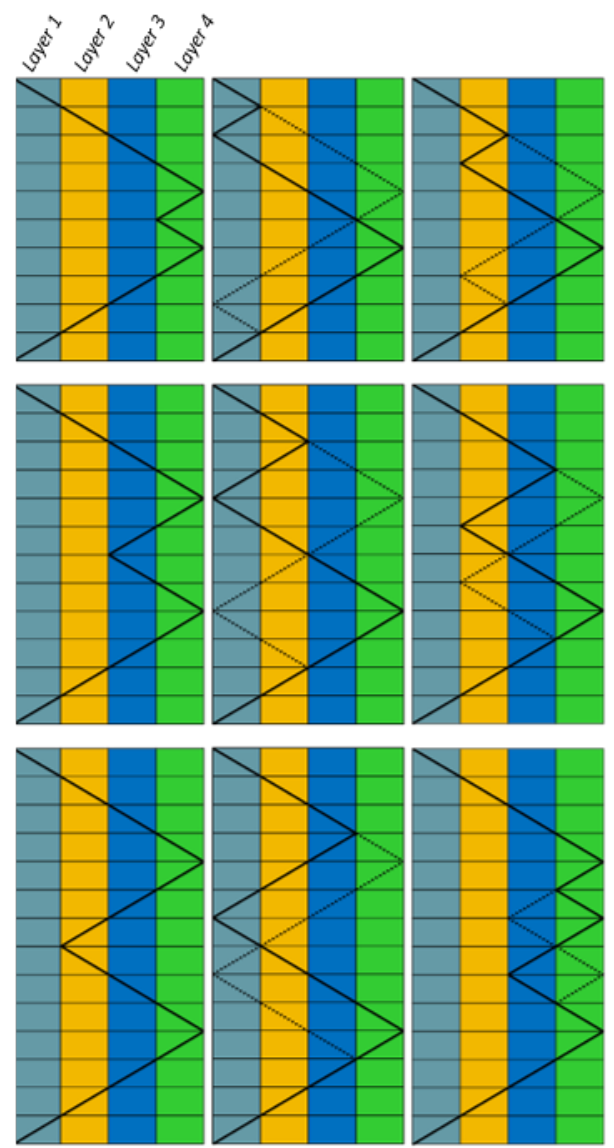

Representation of a portion of the selected optical routes found to be necessary to reconstruct the experimental waveform. 
Doing so, one can therefore calculate a parametric transfer function that only considers a selection of the most contributing routes. Some of these identified optical paths are shown in Figure 8. It is worth to note that the procedure initially dealt with 1,094 distinct paths between $k=1$ and $k=$ 17. However, the identification of the most contributing paths led to a shortlist of 33 routes. Globally, the time needed to reduce the number of optical paths to be considered per pulse subdivision varies from few seconds to few minutes, but remains dictated by solver configuration. Notably, the higher the iteration is, the longer the required time for optical routes minimization is.

Deeper explanation about selection rules can be found in [21]. Ultimately, these 33 selected optical paths were used to reconstruct the previously simulated waveform (Figure 9).

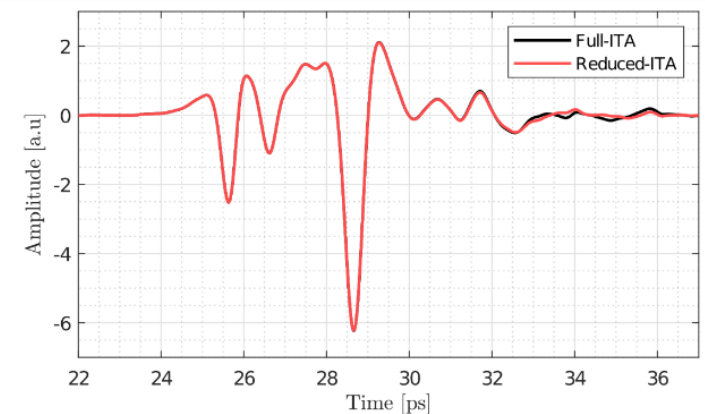

Figure 9: Reconstruction of the signal provided by the restricted selection of optical paths (red) in comparison to the previously reconstructed waveform by means of all propagation routes (black). Divergences appear after 32 ps, where additional subdivided pulses have been deliberately omitted.

This new algorithm is used to understand a stratified material response by terahertz time domain characterization in reflection. The identification of the main optical paths responsible for the reflected signal led to a reduced transfer function that was used to reconstruct almost perfectly the original responsive waveform. The generated parametric transfer function can eventually be implemented in thickness determination algorithms for micrometric coating stack. This algorithm also carries interest to deepen theoretical understanding on the way a responsive terahertz electric field forms upon interacting with a multilayer structure.

\section{Conclusions}

Two real case applications using state of the art apparatus working in the so-called terahertz band were presented. Ultra-Wide Bandwidth FMCW radars are known for their contactless Non-Destructive-Testing capabilities for the aeronautics industry. Here, they proved to be an adequate, compact and sensitive imaging system for remote sensing of inaccessible targets through plane panel thanks to the object visualization and identification as well as alteration and defect detections. Further ongoing works are oriented toward the adequacy of this technique applied to other types of materials. The implementation of a more versatile FMCW radar system together with other optical NDT tools to reach a resourceful imaging system is also under development and is intended to be integrated with an automated positioning system.

For the potential of terahertz time domain measurements on painting thickness, a combination of numerical strategies was described to extract the parameters that describe the best a measured signal arising from a multilayered sample. From simulated reflected waveforms, through Fresnel's equations, an iterative and genetic algorithm then selects the highest contributing optical path (depending on the material response and its thickness) amongst the stack for the generation a reduced transfer function. This new predictive tool is crucial to analyse any deviation for the awaited signal and to identify which layer does not follow the right thickness or dielectric properties.

\section{Acknowledgements}

The authors thank Nouvelle Région d'Aquitaine and BPI France for Athermo project funding. They also thank ATR company for providing real sample for the demonstration. The authors would like to thank OPTIKAN SAS for fruitful discussions.

\section{Supplementary materials}

An animation of the FMCW radar 3D scan for objects detections through the airplane cover is attached to the online version

\section{References}

[1] Cooper KB, Dengler RJ, Llombart N, Bryllert T, Chattopadhyay G, Schlecht E, et al. Penetrating 3-D imaging at 4- and 25-m range using a submillimeterwave radar. IEEE Trans Microw Theory Tech 2008;56:2771-8.

https://doi.org/10.1109/TMTT.2008.2007081.

[2] Cassar Q, Al-Ibadi A, Mavarani L, Hillger P, Grzyb J, MacGrogan G, et al. Pilot study of freshly excised breast tissue response in the $300-600 \mathrm{GHz}$ range. Biomed Opt Express 2018;9:2930. https://doi.org/10.1364/boe.9.002930.

[3] Grootendorst MR, Fitzgerald AJ, Brouwer de Koning SG, Santaolalla A, Portieri A, Van Hemelrijck M, et al. Use of a handheld terahertz pulsed imaging device to differentiate benign and malignant breast tissue. Biomed Opt Express 2017;8:2932. https://doi.org/10.1364/boe.8.002932. 
[4] Cristofani E, Friederich F, Wohnsiedler S, Matheis C, Jonuscheit J, Vandewal $M$, et al. Nondestructive testing potential evaluation of a terahertz frequencymodulated continuous-wave imager for composite materials inspection. Opt Eng 2014;53:031211. https://doi.org/10.1117/1.OE.53.3.031211.

[5] Friederich F, May K, Baccouche B, Matheis C, Bauer $\mathrm{M}$, Jonuscheit $\mathrm{J}$, et al. Terahertz Radome Inspection. Photonics 2018;5:1. https://doi.org/10.3390/photonics5010001.

[6] Dandolo CLK, Guillet J-P, Ma X, Fauquet F, Roux M, Mounaix P. Terahertz frequency modulated continuous wave imaging advanced data processing for art painting analysis. Opt Express 2018;26:5358. https://doi.org/10.1364/oe.26.005358.

[7] Guillet JP, Roux M, Wang K, Ma X, Fauquet F, Balacey $\mathrm{H}$, et al. Art Painting Diagnostic Before Restoration with Terahertz and Millimeter Waves. J Infrared, Millimeter, Terahertz Waves 2017;38:36979. https://doi.org/10.1007/s10762-017-0358-1.

[8] Ellrich F, Bauer M, Schreiner N, Keil A, Pfeiffer T, Klier J, et al. Terahertz Quality Inspection for Automotive and Aviation Industries. J Infrared, Millimeter, Terahertz Waves 2020;41:470-89. https://doi.org/10.1007/s10762-019-00639-4.

[9] Perraud J-B, Guillet J-P, Redon O, Hamdi M, Simoens F, Mounaix P. Shape-from-focus for real-time terahertz 3D imaging. Opt Lett 2019;44:483. https://doi.org/10.1364/OL.44.000483.

[10] Balacey H, Recur B, Perraud JB, Sleiman JB, Guillet JP, Mounaix P. Advanced Processing Sequence for 3D THz Imaging. IEEE Trans Terahertz Sci Technol 2016;6:191-8.

https://doi.org/10.1109/TTHZ.2016.2519263.

[11] Pauli M, Göttel B, Scherr S, Bhutani A, Ayhan S, Winkler W, et al. Miniaturized Millimeter-Wave Radar Sensor for High-Accuracy Applications. IEEE Trans Microw Theory Tech 2017;65:1707-15. https://doi.org/10.1109/TMTT.2017.2677910.

[12] Wong TM, Kahl M, Haring Bolívar P, Kolb A. Computational Image Enhancement for Frequency Modulated Continuous Wave (FMCW) THz Image. J Infrared, Millimeter, Terahertz Waves 2019;40:775800. https://doi.org/10.1007/s10762-019-00609-w.

[13] Ding J, Kahl M, Loffeld O, Bolívar P. THz 3-D Image Formation Using SAR Techniques: Simulation,ProcessingandExperimentalResults. Proc Trans TERAHERTZ Sci Technol 2013;3:284-7.
https://doi.org/10.1109/ULTSYM.2007.82.

[14] Perraud JB, Obaton AF, Bou-Sleiman J, Recur B, Balacey H, Darracq F, et al. Terahertz imaging and tomography as efficient instruments for testing polymer additive manufacturing objects. Appl Opt 2016;55:3462. https://doi.org/10.1364/ao.55.003462.

[15] Schreiner NS, Sauer-Greff W, Urbansky R, Friedcrich F. All-Electronic High-Resolution Terahertz Thickness Measurements. Int Conf Infrared, Millimeter, Terahertz Waves, IRMMW-THz 2018;2018-Septe:2-3.

https://doi.org/10.1109/IRMMW-THz.2018.8510060.

[16] Roman C, Ichim O, Sarger L, Vigneras V, Mounaix P. Terahertz dielectric characterisation of polymethacrylimide rigid foam: The perfect sheer plate? Electron Lett 2004;40:1167-9. https://doi.org/10.1049/el:20045754.

[17] Krimi S, Klier J, Jonuscheit J, Freymann G Von, Urbansky R, Beigang R. Highly accurate thickness measurement of multi-layered automotive paints using terahertz technology. Appl Phys Lett 2016;109. https://doi.org/10.1063/1.4955407.

[18] Picot M, Ballacey H, Guillet JP, Cassar Q, Mounaix P. Terahertz Paint Thickness Measurements : from lab to automotive and aeronautics industry. APNDT2017 2017:1-8.

[19] van Mechelen JLM, Kuzmenko AB, Merbold H. Stratified dispersive model for material characterization using terahertz time-domain spectroscopy. Opt Lett 2014;39:3853. https://doi.org/10.1364/ol.39.003853.

[20] Cassar, Q. Koch-Dandolo, C. L. Guillet, J. P. Roux, M. Fauquet, F. Perraud, J. B. Mounaix, P.. "Characterization of Varnish Ageing and its Consequences on Terahertz Imagery: Demonstration on a Painting Presumed of the French Renaissance." Journal of Infrared, Millimeter, and Terahertz Waves (2020): 1-11.

[21] Goodfellow, Ian and Bengio, Yoshua and Courville A. Deep learning. MIT press; 2016.

[22] Cassar Q, Chopard A, Fauquet F, Guillet J-P, Pan M, Perraud J-B, et al. Iterative Tree Algorithm to Evaluate Terahertz Signal Contribution of Specific Optical Paths within Multi-Layered Materials. IEEE Trans Terahertz Sci Technol 2019;9:684-94. https://doi.org/10.1109/tthz.2019.2937208. 